REVISTA MATEMÄTICA de la

Universidad Complutense de Madrid

Volumen 6, número 1; 1993.

http://dx.doi.org/10.5209/rev_REMA.1993.v6.n1.17860

\title{
On the Behaviour of Generalized Solutions to Genuinly Nonlinear First Order Equations for Small and Large Values of Time
}

\author{
YU G. RYKOV
}

ABSTRACT. The paper deals with the asymptotic behaviour of generalized solutions to nonlinear first order equations. With the aid of explicit variational representation one studies the decrease of solutions for a large time. And for the small time an asymptotics of the perturbation's front is calculated.

\section{INTRODUCTION}

This paper investigates generalized solutions (briefly:g.s.) of the equation

$$
L u \equiv u_{t}+f\left(t, x, u_{x}\right)+g(t, x, u)=H(t, x),(t, x) \in \mathbf{R}_{+} \times \mathbf{R}
$$

with initial data

$$
u(0, x)=u_{0}(x), \quad x \in \mathbf{R}
$$

Here $f \in \mathrm{C}^{3}, g \in \mathrm{C}, g(t, x, u)$ satisfies local Hölder condition in $u \in \mathbf{R}_{+}$ 
with exponent $n>0$ and has three continuous derivatives in $u \in \mathbb{R}_{+}$, $f(t, x, 0)=g(t, x, 0)=0, g(t, x, u)$ monotonically nondecreases in $u, f_{p p}(t, x, p) \geq \mu>0$, $H(t, x)$ is a measurable function bounded for bounded $t ; u_{0}(x) \in \mathbf{W}_{\infty}^{1}(\mathbb{R})$, $u_{0}(x) \geq 0$.

The Cauchy problem for (1.1) was considered in [2], [8]-[10]. The papers [11], [3]-[7] are devoted to the case of $f$ nonconvex with respect to $u_{x}$. Further references may be found in the cited articles.

In $\$ 2$ we deal with the decreasing of g.s. to $(1.1),(1.2)$ as $t$ converges to $+\infty$ in the case

$$
f\left(t_{r} x, u_{x}\right)=f\left(u_{x}\right), g\left(t_{r} x, u\right) \equiv 0, \lim _{|x| \rightarrow+\infty} u_{0}(x)=0, H\left(t_{r} x\right) \equiv 0 .
$$

In [8] for this case stabilization to inf $u_{0}(x)$ was proved and the rate of the stabilization was estimated. Similar results but in the case of nonconvex $f$ are presented in [1]. Our results are based on explicit formula for the g.s. of the problem (1.1), (1.2) obtained in [9]. Some function $v(t, x)$ satisfying (1.1) in the generalized sense will be found such that for every $u_{0}(x)$ with compact support and nonsmooth at the points of the support boundary the corresponding g.s. of (1.1), (1.2) identically equals $v(t, x)$ beginning from some fixed time. If $u_{0}(x)$ is smooth then the $\mathrm{g}$.s. tends to $v(t, x)$ as $t$ converges to $+\infty$ and the rate of convergence will be estimated.

In case $u_{0}(x)$ does not possess compact support but tends to zero as $|x| \rightarrow+\infty$ the rate of g.s. decreasing also will be estimated.

$\$ 3$ is devoted to the support behaviour of g.s. to (1.1), (1.2) for small values of $t$ provided $u_{0}(x)$ has compact support. For example, consider the equation

$$
u_{\ell}+f\left(u_{x}\right)+N u_{x}+K\left\{\left.u\right|^{n-1} u, \quad(t, x) \in \mathbb{R}_{+} \times \mathbf{R}\right.
$$

where $f(0)=f^{\prime}(0)=0 f^{\prime \prime}>0, N \geq 0, K \geq 0,0<n<1$.

Suppose $u_{0}(x)=P(1-x)^{\alpha}$ for $1-\varepsilon \leq x \leq 1, u_{0}(x) \equiv 0$ for $x>1$, where $\alpha \geq 1$, $P>0, \varepsilon>0$. For this particular case as a corollary of the results contained 
in $\S 3$ one obtains necessary and sufficient conditions for the support of the g.s. of (1.1), (1.2) to expand in the left or the right direction.

Definition 1.1. A function $u(t, x)$ Lipschitz continuous in $[0, T] \times \boldsymbol{R}$, $T>0$, is called a g.s. of the problem (1.1), (1.2) if:

1) $u(t, x)$ satisfies (1.1) almost everywhere in $\boldsymbol{R}_{+} \times \boldsymbol{R}$ and takes initial values (1.2);

2) for every $l \neq 0$ in each rectangle $(0, T] \times[-R, R], T>0, R>0$, the following inequality is fulfilled

$$
u(t, x+l)-2 u(t, x)+u(t, x-l) \leq \lambda(t, R) l^{2},
$$

where $\lambda(t, R) \geq 0$ is defined for $t \in(0, T]$ and $\lambda(t, R)<\lambda_{\delta}<+\infty$ for $0<\delta \leq t \leq T$.

The existence theorem for the problem (1.1), (1.2) one can prove by analogy with [8].

Theorem 1.1. Suppose $v(t, x) \in W_{\infty}^{l}, w(t, x) \in W_{\infty}^{I}$ in each strip $[0, T] \times \boldsymbol{R}, T>0$. Suppose $v(t, x)$ is a g.s. of the equation $L v=h_{1}(t, x)$ with data $v(0, x)=v_{0}(x) \in W_{\infty}^{l}(\boldsymbol{R})$, and $w(t, x)$ is a g.s. of the equation $L w=h_{2}(t, x)$ with data $w(0, x)=w_{0}(x) \in W_{\text {od }}^{l}(\boldsymbol{R})$, where $h_{f}(t, x), h_{2}(t, x)$ are measurable functions bounded for bounded t. Suppose $h_{l}(t, x) \leq h_{2}(t, x)$ in $\boldsymbol{R}_{+} \times \mathbb{R}$ and $v_{0}(x) \leq w_{0}(x)$ in $\boldsymbol{R}$. Then $v(t, x) \leq w(t, x)$ in $\boldsymbol{R}_{+} \times \boldsymbol{R}$.

Due to monotonicity of $g(t, x, u)$ in $u$ the proof of this theorem is analogous to [8]. It follows from the Theorem 1.1 that the g.s. of (1.1), (1.2) is unique.

Below $u(t, x)$ denotes the g.s. of the problem (1.1), (1.2) with $H(t, x) \equiv 0$. 


\section{THE BEHAVIOUR OF GENERALIZED SOLUTIONS AS t CONVERGES TO $+\infty$}

Suppose $f\left(t, x, u_{x}\right) \equiv f\left(u_{x}\right), g(t, x, u) \equiv 0, f^{\prime}(0)=0, f \in C^{4}(\mathbf{R})$. Under these conditions (see [9]) the g.s. of the problem $(1.1),(1.2)$ with $H(t, x) \equiv 0$ is given by the formula

$$
u(t, x)=\inf _{q \in \mathbf{R}} G\left(t, x, q, u_{0}(q)\right), G\left(t, x, q, u_{0}(q)\right) \equiv u_{0}(q)+t \Phi((x-q) / t),
$$

where $\Phi(q)$ is Legendre transformation of the function $f(q), \Phi(q)=f^{-1}(q) q$ - $f \circ f^{-1}(q) \geq 0$, because of the convexity of $f(q)$. Notice, that $\Phi^{\prime}(q)=f^{-1}(q)$.

Let us fulfil some auxiliary research and study the roots of the equation (with respect to $q$ )

$$
G_{q}=u_{0}^{\prime}(q)-f^{\prime-1}((x-q) / t)=0, \quad q \in[a, b]
$$

where $>0, x \in \mathbb{R}$ are fixed.

Lemma 2.1. Suppose $u_{0}(t, x)$ is monotone in $[a, b], u_{0} \in C([a, b])$. Then if $[a, b]$ does not contain zero roots for $u_{0}^{\prime}(q)=0$ there exists such $t_{0}$ that for $t>t_{0}$ the segment $[a, b]$ does not contain roots of $(2.2)$; if $q_{*} \in[a, b]$, $u_{0}^{\prime}\left(q_{*}\right)=0$ and $q_{*}$ is the unique root for $u_{0}^{\prime}(q)=0$ then there exists such $t_{1}$ that for $t>t$, the equation (2.2) has only one root tending to $q_{*}$ as $t \rightarrow+\infty$.

Proof. The equation (2.2) is equivalent to the following

$$
f^{\prime} \circ u_{0}^{\prime}(q)=(x-q) / t
$$

The left hand side of (2.3) equals zero if and only if $u_{0}^{\prime}(q)=0$. The right hand side of (2.3) for fixed $x$ tends to zero as $t \rightarrow+\infty$ uniformly with respect to $q \in[a, b]$. Now, the statements of Lemma 2.1 easily follow. 
Lemma 2.2. Suppose supp $u_{0}(x) \subset[a, b]$, Then supp $u(t, x) \subset \boldsymbol{R}_{+} \times[a, b]$.

Proof. Suppose $x \notin[a, b], t \in \mathbf{R}_{+}$. Let us take $q=x$, then $G\left(t, x, x, u_{0}(x)\right)$ $=u_{0}(x)=0$. Our result follows from (2.1).

Let us introduce the notation

$$
v(t, x)=\min \left[G\left(t, x, 0, u_{0}(0)\right), G\left(t, x, 1, u_{0}(1)\right)\right] .
$$

Theorem 2.1. Suppose the following conditions hold:

1) supp $u_{0}(x)=[0,1]$

2) $u_{0}(x) \geq l_{1}(x), l_{i}(x) \in W_{\infty}^{\prime}(\boldsymbol{R})$ and $l_{i}(x)>0, l_{1}(x \pm 0) \neq 0$ in $(0,1)$;

3) $u_{0}(x)=l_{l}(x)$ for $|x|<\varepsilon$ and for $|x-1|<\varepsilon$ for some $\varepsilon>0$;

4) $l_{1}(x) \sim a x^{\alpha}, l_{l}^{\prime}(x)-\alpha a x^{\alpha-l}$ as $x \rightarrow+0$;

5) $l_{1}(x)-a(1-x)^{\alpha}, l_{l}(x)-\alpha a(1-x)^{\alpha-1}$ as $x \rightarrow 1-0$;

here $a=$ const $>0, \alpha=$ const $>1$

Then $t^{\alpha /(\alpha-I)}[v(t, x)-u(t, x)] \rightarrow B(x)$ as $t \rightarrow+\infty$ for every $x \in \boldsymbol{R}$, where $B(x)=0$ for $x \notin(0,1)$ and

$$
B(x)=(\alpha-1) a^{-1 /(\alpha-1)}\left[\alpha f^{\prime \prime}(0)\right]^{-\alpha /(\alpha-1)} \min \left\{x^{\alpha /(\alpha-1)},(1-x)^{\alpha /(\alpha-1)\}}\right.
$$

for $x \in(0,1)$.

Proof. It follows from the conditions of Theorem 2.1 that $G\left(t, x, q, u_{0}(q)\right) \geq G\left(t, x, q, I_{1}(q)\right)$. By virtue of Lemma $2.1 G\left(t, x, q, I_{1}(q)\right)$ as a function of $q$ can have a local minimum at $\varepsilon$-neighbourhoods of the points $q=0$ and $q=1$ provided $t$ is sufficiently large. But there $u_{0}(q)=l_{1}(q)$. It is easy to see that $G\left(t, x, q, u_{0}(q)\right)>0, q \in(0,1)$ because of $u_{0}(q)>0$ for $q \in(0,1)$. So global minimum with respect to $q$ of the function $G\left(t, x, q, u_{0}(q)\right)$ coincides with the smallest of the values $G(t, x, 0,0)$, $G(t, x, 1,0), G\left(t, x q_{0}, u_{0}\left(q_{0}\right)\right), G\left(t, x, q_{1}, u_{0}\left(q_{1}\right)\right)$, where $q_{0}=q_{0}(t, x), q_{1}=q_{1}(t, x)$ are the roots of (2.3) tending to 0 and 1 respectively as $t \rightarrow+\infty$. 
Notice, that $G(t, x, 0,0)=t \Phi(x / t), G(t, x, 1,0)=t \Phi((x-1) / t)$. It is easy to see that for sufficiently large $t$ the following inequalities are true:

$$
\begin{array}{ll}
G(t, x, 0,0) \leq G(t, x, 1,0) & \text { for } 0 \leq x \leq 1 / 2 \\
G(t, x, 0,0) \geq G(t, x, 1,0) & \text { for } 1 / 2 \leq x \leq 1 .
\end{array} \quad \text { and }
$$

Hence, provided $t$ is large enough we obtain

$$
\begin{array}{ll}
G\left(t, x, q_{0}, u_{0}\left(q_{0}\right)\right) \leq G\left(t, x, q_{1}, u_{0}\left(q_{1}\right)\right) & \text { for } 0 \leq x<1 / 2 \\
G\left(t, x, q_{0}, u_{0}\left(q_{0}\right)\right) \geq G\left(t, x, q_{1}, u_{0}\left(q_{1}\right)\right) & \text { for } 1 / 2<x \leq 1 .
\end{array} \quad \text { and }
$$

Let us seek $q_{0}(t, x)$ in the form $q_{0}(t, x)=b_{0}(x) t^{-\beta}+o\left(t^{-\beta}\right)$, where $b_{0} \in \mathbf{C}(\mathbf{R})$, $\beta>0$. Substituting $q_{0}(t, x)$ into (2.2) one gets $\alpha a q_{0}(t, x)^{\alpha-1}+o\left(q_{0}(t, x)^{\alpha-1}\right)=$ $\Phi^{\prime}\left(\left(x-q_{0}(t, x)\right) / t\right), \alpha a b_{0}(x)^{\alpha-1} t^{-\beta(\alpha-1)}+o\left(t^{-\beta(\alpha-1)}\right)=\Phi^{\prime \prime}(0)\left(x-q_{0}(t, x)\right) / t+o(1 / t)$. Hence $\beta=1 /(\alpha-1)$ and equating coefficients at $t^{-1}$ one finds $a \alpha b_{0}^{\alpha-1}(x)=$ $x \Phi^{\prime \prime}(0)$. Further, $G(t, x, 0,0)-G\left(t, x, q_{0}(t, x), u_{0}\left(q_{0}(t, x)\right)\right)=t \Phi(x / t)-t \Phi((x-$ $\left.\left.q_{0}(t, x)\right) / t\right)-u_{0}\left(q_{0}(t, x)\right)=t\left[\Phi^{\prime}\left(\left(x-q_{0}(t, x)\right) / t\right) q_{0}(t, x) / t+\int_{\left(x-q_{0}(t, x)\right) / t}^{x / t} \Phi^{\prime \prime}(\xi)(x / t-\xi) d \xi\right]-$ $a q_{0}(t, x)^{\alpha}+o\left(t^{-\alpha /(\alpha-1)}\right)$. One estimates $t\left|\int_{\left(x-q_{0}(t, x)\right) / t}^{x / x} \Phi^{-"}(\xi)(x / t-\xi) d \xi\right| \leq$ const $t_{(x-q \alpha(t, x)) / t}^{x / t}|x / t-\xi| d \xi \leq$ const $q_{0}(t, x)^{2} / t=\alpha\left(t^{-\beta-1}\right)=$ o $\left(t^{\alpha /(\alpha-1)}\right)$. Besides, $\Phi^{\prime}((x-$ $\left.\left.q_{0}(t, x)\right) / t\right)=\Phi^{\prime \prime}(0)\left(x-q_{0}(t, x)\right) / t+o(1 / t)$.

Thus, $G(t, x, 0,0)-G\left(t, x, q_{0}(t, x), u_{0}\left(q_{0}(t, x)\right)\right)=\Phi^{\prime \prime}(0) x q_{0}(t, x) / t-$ $a b_{0}(x)^{\alpha_{1}-\alpha /(\alpha-1)}+\mathrm{o}\left(t^{-\alpha /(\alpha-1)}\right)=\left[\Phi^{\prime \prime}(0) x b_{0}(x)-a b_{0}(x)^{\alpha}\right] t^{-\alpha /(\alpha-1)}+\mathrm{o}\left(t^{-\alpha /(\alpha-1)}\right)$. Now, taking into account that $\Phi^{\prime \prime}(0)=1 / f^{\prime \prime}\left(f^{\prime \prime}(0)\right)=1 / f^{\prime \prime}(0)$, one finds $G(t, x, 0,0)$ $G\left(t, x, q_{0}(t, x), u_{0}\left(q_{0}(t, x)\right)\right)=b_{0}(x) x(1-1 / \alpha) t^{-a /(\alpha-1)} / f^{\prime \prime}(0)+o\left(t^{\text {oul(to-1) }}\right)$. The first term of the right hand side is positive for $x>0$.

By analogy one can prove the equality $G(t, x, 1,0)-G\left(t, x, q_{1}(t, x)\right.$, $\left.u_{0}\left(q_{1}(t, x)\right)\right)=t^{-\alpha / \alpha-1)} b_{0}(1-x)(1-x)(1-1 / \alpha) / f^{\prime \prime}(0)+o\left(t^{-\alpha /(\alpha-1)}\right)$. Here the first term of the right hand side is positive for $x<1$.

Comparing the last two formulas one proves the statement of Theorem 2.1 for $x \in(0,1)$. For other $x B(x)=0$ by virtue of Lemma 2.2.

Theorem 2.2. Suppose the following conditions hold:

1) $\operatorname{supp} u_{0}(x)=[0,1]$; 
2) $u_{0}(x) \geq l_{2}(x)$ in $(0,1), l_{2}(x)=M x / \varepsilon$ for $0<x \leq \varepsilon$, $l_{2}(x)=M(1-x) /(1-\varepsilon)$ for $\varepsilon \leq x<1, M>0,1>\varepsilon>0$;

3) $\Phi(s) \leq M s / \varepsilon$ for $0 \leq s \leq s_{0}(M, \varepsilon), \Phi(-s) \leq M s /(1-\varepsilon)$ for $0 \leq s \leq s_{l}(M, \varepsilon)$, where $\Phi$ is the Legendre transformation of $f$.

Then $u\left(t_{x} x\right)=v(t, x)$ for $t \geq T=\max \left[-1 / f^{\prime}(-M /(1-\varepsilon)), 1 / f^{\prime}(M / \varepsilon)\right.$, $\left.s_{0}(M, \varepsilon)^{-i}, s_{l}(M, \varepsilon)^{-i}\right]$.

Proof. Let us investigate infimum (with respect to $q$ ) of the function $G\left(t, x, q, l_{2}(q)\right)$. Because of Lemma 2.2 one may consider only $0<x<1$. We are interested in the values of the function $G$ at the points $q$, where either $G_{q}=0$ or $G$ is not smooth. $G$ is not smooth at three points: $0, \varepsilon, 1$.

If $q<0$ then $G_{q}=0$ only at $q=x$; if $0<q<\varepsilon$ then $G_{q}=0$ at $q=x-t f^{f}(M / \varepsilon)$; if $\varepsilon<q<1$ then $G_{q}=0$ at $q=x-t f^{\prime}(-M /(1-\varepsilon))$; if $q>1$ then $G_{q}=0$ only at $q=x$.

Thus, the global minimum on the funcion $G\left(t, x, q, l_{2}(q)\right)$ with respect to $q$ coincides with the smallest of the values:

$\omega_{1} \equiv t \Phi(x / t), \quad \omega_{2} \equiv t \Phi((x-1) / t), \quad \omega_{3} \equiv M+t \Phi((x-\varepsilon) / t), \quad \omega_{4} \equiv l_{2}(x), \omega_{5} \equiv$ $l_{2}\left(x-t f^{\prime}(M / \varepsilon)\right)+t \Phi \circ f^{\prime}(M / \varepsilon), \omega_{6} \equiv l_{2}\left(x-t f^{\prime \prime}(-M /(1-\varepsilon))\right)+t \Phi \circ f^{\prime}(-M /(1-\varepsilon))$.

For $0<x<1, x<t f^{\prime \prime}(M / \varepsilon), x>1+t f^{\prime}(-M /(1-\varepsilon))$ the following inequalities are true: $\omega_{1} \leq \omega_{5}, \omega_{2} \leq \omega_{6}$. Because of assumption 3) $\min \left(\omega_{1}, \omega_{2}\right) \leq \omega_{4}$ for $t \geq T$. Further $\omega_{3}>M$ in consequence to $\Phi>0$. Thus, it sufficies to find $\min \left(\omega_{1}, \omega_{2}\right)$ for $t \geq T$. But $G\left(t, x, q, u_{0}(q)\right) \geq G\left(t, x, q, l_{2}(q)\right)$ for every $q$. Hence

$$
\inf _{q \in \mathbb{R}} G\left(t, x, q, u_{0}(q)\right)=\inf _{q \in \mathbb{R}} G\left(t, x, q, l_{2}(q)\right)=\min \{t \Phi(x / t), \tau \Phi((x-1) / t)\}=v(t, x) \text { for } t \geq T \text {. }
$$

Remark 2.1. It follows from the theorems $2.1,2.2$ that $u(t, x)$ tends to zero as $t \rightarrow+\infty$ in case $u_{0}(x)$ is not smooth at the points 0 and 1 slower than in case $u_{0}(x)$ is smooth there. But this difference presents only in the second term of the asymptotics as $t \rightarrow+\infty$. 
Theorem 2.3. Suppose the following conditions hold:

1) $u_{0}(x)>0, x \in \boldsymbol{R}$

2) $u_{0}(x) \geq l_{3}(x), l_{3} \in W_{\infty}^{\prime}(\boldsymbol{R}), l_{3}(x)>0, l_{3}(x \pm 0) \neq 0$ for $x \in \boldsymbol{R}$;

3) $u_{0}(x)=l_{3}(x)$ for $|x|$ sufficiently large;

4) $l_{3}(x) \sim C_{J}|x|^{-\beta}+C_{2}|x|^{-\beta-1}$, $l_{3}(x)--\beta C_{1}|x|^{-\beta-I} \operatorname{sign} x-(\beta+1) C_{2}|x|^{-\beta-2} \operatorname{sign} x$, $|x| \rightarrow+\infty, C_{1}>0, C_{2}>0, \beta>0$.

Then $\left.t^{(\beta+1) /(\beta+2)} u(t, x) \quad-\quad C_{I}^{2 /(\beta+2)}(\beta+2) 2^{-1} / \Phi^{\prime \prime}(0) / \beta\right]^{\beta /(\beta+2)} t^{l /(\beta+2)} \rightarrow$ $\phi^{\prime \prime}(0)^{(\beta+1) /(\beta+2)}\left(\beta C_{l}\right)^{l /(\beta+2)}\left[C_{2} /\left(\beta C_{l}\right)-|x|\right]$ as $t \rightarrow+\infty$ for every $x \in \boldsymbol{R}$.

Proof. By analogy with the proof of Theorem 2.1 it sufficies to find the minimum with respect to $q$ of the function $G\left(t, x, q, l_{3}(q)\right)$. The equation (2.3) with respect to $q$ has two roots $q_{+}(t, x)$ and $q .(t, x)$ tending to $+\infty$ and $-\infty$ respectively as $t \rightarrow+\infty$. Let us seek these roots in the form

$$
q_{ \pm}(t, x)= \pm a_{1} t^{\gamma}\left(1+a_{2}(x) t^{-\delta}+o\left(t^{-\delta}\right)\right) \text {, }
$$

where $a_{1}=$ const $>0, a_{2} \in \mathrm{C}(\mathbf{R}), \gamma>0, \delta>0$.

Let us consider $q_{+}$. Applying Taylor's formula with the remainder term in Peano's form one gets

$\dot{l_{3}}\left(q_{+}(t, x)\right)=-\beta C_{1} a_{1}^{-(\beta+1)} t^{-\alpha(\beta+1)}\left(1+a_{2}(x) t^{\delta}+\mathrm{o}\left(t^{\delta}\right)\right)^{-\beta-1}-$ $\left.(\beta+1) C_{2} a_{1}^{-(\beta+2)}\right) t^{-\gamma(\beta+2)}\left(1+a_{2}(x) t^{-\delta}+o\left(t^{-\delta}\right)\right)^{-\beta-2}+o\left(t^{-\gamma(\beta+2)}\right) ;$ $\Phi^{\prime}\left(\left(x-q_{+}(t, x)\right) / t\right)=\Phi^{\prime \prime}(0)\left[x / t-a_{1} t^{\gamma-1}\left(1+a_{2}(x) t^{\delta}+o\left(t^{\delta}\right)\right)\right]+$ $\Phi^{\prime \prime \prime}(0) 2^{-1}\left[x / t-a_{1} t^{\gamma-1}\left(1+a_{2}(x) t^{-\delta}+o\left(t^{-\delta}\right)\right)\right]^{2}+o\left(t^{2(1-\gamma)}\right)$.

Substituting these expresions into (2.3) and comparing the terms with the lowest absolute value of powers of $t$ one obtains

$$
\gamma=1 /(\beta+2), a_{1}^{\beta+2}=\beta C_{1} / \Phi^{\prime \prime}(0) \text {. }
$$

If $\delta>\gamma$ then $-\gamma(\beta+1)-\delta<-1$ and the supplementary condition on $a_{1}$ will arise: $(\beta+1) C_{2} a_{1}^{-(\beta+2)}=x \Phi^{\prime \prime}(0)$ which is in the contrary with the former. 
If $\delta<\gamma$ then $-\gamma(\beta+1)-\delta>-1$ and $-\gamma(\beta+1)-\delta=-1+\gamma-\delta$; besides $-a_{1} a_{2}(x) \Phi^{\prime \prime}(0)=\beta(\beta+1) C_{1} a_{1}^{-(\beta+1)} a_{2}(x)$. It is impossible because the left hand the right hand quantities are of opposite signs. that is

Hence $\delta=\gamma$ and $a_{1}^{-(\beta+2)}\left[C_{\mathrm{l}} \beta(\beta+1) a_{1} a_{2}(x)-(\beta+1) C_{2}\right]=\Phi^{\prime \prime}(0)\left(x-a_{1} a_{2}(x)\right)$,

$$
a_{1}^{(\beta+2)}=\beta C_{1} / \Phi^{\prime \prime}(0), a_{2}(x)=\left[\beta C_{1} x+C_{2}(\beta+1)\right] /\left[\beta(\beta+2) C_{1} a_{1}\right]
$$

Using formulas (2.4) one gets

$G\left(t, x, q_{+}(t, x), l_{3}\left(q_{+}(t, x)\right)\right)=C_{1} q_{+}(t, x)^{-\beta}+C_{2} q_{+}(t, x)^{-\beta \cdot 1}+$ $\mathrm{o}\left(t^{-(\beta+1) /(\beta+2)}\right)+t\left[2^{-1} \Phi^{\prime \prime}(0)\left(\left(x-q_{+}(t, x)\right) / t\right)^{2}+6^{-1} \phi^{\prime \prime}(0) \times\right.$ $\left.\left(\left(x-q_{+}(t, x)\right) / t\right)^{3}+\mathrm{o}\left(t^{3(\beta+1) /(\beta+2)}\right)\right]=\left[C_{1} a_{1}^{-\beta}+2^{-1} \phi^{\prime \prime}(0) a_{1}^{2}\right] \times$ $t^{-\beta /(\beta+2)}+\left[\Phi^{\prime \prime}(0) a_{1}^{2} a_{2}(x)-\beta C_{1} a_{1}^{-\beta} a_{2}(x)+C_{2} a_{1}^{-\beta-1}-\Phi^{\prime \prime}(0) a_{1} x\right] \times$ $t^{(\beta+1) /(\beta+2)}+\mathrm{o}\left(t^{(\beta+1) /(\beta+2)}\right)=a_{1}^{2}(1 / \beta+1 / 2) \Phi^{\prime \prime}(0) t^{-\beta /(\beta+2)}+$ $a_{1} \Phi^{\prime \prime}(0)\left[C_{2} /\left(\beta C_{1}\right)-x\right] t^{-(\beta+1) /(\beta+2)}+\mathrm{o}\left(t^{-(\beta+1) /(\beta+2)}\right)$.

By analogy one finds

$G\left(t, x, q_{-}(t, x), l_{3}\left(q_{-}(t, x)\right)\right)=a_{1}^{2}(1 / \beta+1 / 2) \Phi^{\prime \prime}(0) t^{\beta /(\beta+2)}+$ $a_{1} \Phi^{\prime \prime}(0)\left[C_{2} /\left(\beta C_{1}\right)+x\right] t^{(\beta+1))(\beta+2)}+\mathrm{o}\left(t^{-(\beta+1) /(\beta+2)}\right)$.

It is easy to see that

$u(t, x)=\min \left[G\left(t, x, q_{+}(t, x), l_{3}\left(q_{+}(t, x)\right)\right), G\left(t, x, q .(t, x), l_{3}(q .(t, x))\right)\right]$

and get the statement of Theorem 2.3.

Theorem 2.4. Suppose assumptions I), 2) of the Theorem 2.3 hold and assumptions 3), 4) hold only for $x<0$; besides $f^{\prime}(0)>0$. Then (independently of the behaviour of $u_{0}(x)$ as $x \rightarrow+\infty$ ) the following relation is valid

$$
u(t, x) t^{\beta+1}-C_{1}\left(f^{\prime}(0)\right)^{-\beta} t \rightarrow\left(f^{\prime}(0)\right)^{-\beta-1}\left(C_{2}+\beta C_{1} x\right)
$$

as $t \rightarrow+\infty$, for every $x \in \boldsymbol{R}$. 
Proof. In this case for large $t$ equation (2.3) has only one unbounded root $q_{-}(t, x) \rightarrow-\infty$ as $t \rightarrow+\infty$. Other roots, if they exist, tend to finite limits. It sufficies to establish asymptotics only for the function $p(t, x) \equiv$ $G\left(t, x, q_{-}(t, x), l_{3}(q(t, x))\right)$ as $t \rightarrow+\infty$ because $G\left(t, x, q_{-}(t, x), u_{0}(q-(t, x))\right)$ tends to zero as $t \rightarrow+\infty$.

Let us seek $q .(t, x)$ in the form

$$
q_{-}(t, x)=-a_{1} t^{\gamma}\left(1+a_{2}(x) t^{-\delta}+a_{3} t^{-\Delta}+o\left(t^{-\Delta}\right)\right),
$$

where $a_{1}=$ const $>0, a_{2} \in \mathrm{C}(\mathbb{R}), a_{3}=$ const, $\gamma>0, \Delta>\delta>0$. Then $l_{3}(q(t, x))$ $=\beta C_{1} a_{1}^{-\beta-1} t^{-\gamma(\beta+1)}\left[1+a_{2}(x) t^{-\delta}+a_{3} t^{-\Delta}+o\left(t^{-\alpha}\right)\right]^{-\beta-1}+C_{2}(\beta+1) a_{1}^{-\beta-2} t^{-\gamma \beta+2)}\left[1+a_{2}(x) t^{-\delta}+a_{3} t^{-\Delta}\right.$ $\left.+o\left(t^{-\Delta}\right)\right]^{-\beta-2}+o\left(t^{-\gamma(\beta+2)}\right)$. Now we have $u_{0}^{\prime}(q(t, x)) \rightarrow 0$ as $t \rightarrow+\infty$. It follows from (2.3) that $\left(x-q_{-}(t, x)\right) / t$ tends to $b=$ const as $t \rightarrow+\infty$ and $\Phi^{\prime}(b)=0$ so $\Phi(b)=0$. However, $(x-q(t, x)) / t=x / t+a_{1} t^{\gamma-1}\left(1+a_{2}(x) t^{-\delta}+a_{3} t^{-\Delta}+o\left(t^{-\Delta}\right)\right)$. So $\gamma=1$, $a_{1}=b$. Further,

$\Phi^{\prime}\left(\left(x-q_{-}(t, x)\right) / t\right)=\Phi^{\prime \prime}\left(a_{1}\right)\left[x / t+a_{1} a_{2}(x) t^{-\delta}+a_{1} a_{3} t^{-\Delta}+\mathrm{o}\left(t^{-\Delta}\right)\right]+$ $2^{-1} \Phi^{\prime \prime}\left(a_{1}\right)\left[x / t+a_{1} a_{2}(x) t^{-\delta}+a_{1} a_{3} t^{-\Delta}+o\left(t^{-\Delta}\right)\right]^{2}+o\left(t^{-2 m i n(1, \Delta)}\right)$.

Substituting $\Phi^{\prime}$ and $l_{3}^{\prime}$ into (2.3) one obtains $\delta=1, a_{2}(x)=-x / a_{1}$; further, $\Delta=\beta+1, \beta C_{1} a_{1}^{-\beta-1}=a_{1} a_{3} \Phi^{\prime \prime}\left(a_{1}\right)$. Thus,

$$
\begin{aligned}
& p(t, x)=l_{3}(q-(t, x))+t \Phi((x-q(t, x)) / t)=C_{1} a_{1}^{-\beta} t^{-\beta}\left(1+a_{2}(x) t^{-1}+\right. \\
& \left.a_{3} t^{-\beta-1}+o\left(t^{-\beta-1}\right)\right)^{-\beta}+C_{2} a_{1}^{-\beta-1} t^{-\beta-1}\left(1+a_{2}(x) t^{-1}+a_{3} t^{-\beta-1}+o\left(t^{\beta-1}\right)\right)^{-\beta-1}+ \\
& o\left(t^{-\beta-1}\right)+t\left[2^{-1} \Phi^{\prime \prime}\left(a_{1}\right)\left(a_{1} a_{3} t^{-\beta-1}+o\left(t^{-\beta-1}\right)\right)^{2}\right]=C_{1} a_{1}^{-\beta} t^{-\beta}+ \\
& \left(C_{2} a_{1}^{-\beta-1}-C_{1} a_{1}^{-\beta} \beta a_{2}(x)\right) t^{-\beta-1}+o\left(t^{-\beta-1}\right) .
\end{aligned}
$$

Substituting $a_{2}(x)$ one gets the required asymptotics.

\section{THE BEHAVIOUR OF GENERALIZED SOLUTIONS FOR SMALL $t$}

In this paragraph we consider the equation (1.1) in the form

$$
L_{1} u \equiv u_{r}+f_{1}\left(t, x, u_{x}\right)+B u_{x}+g(t, x, u)=0,
$$

where $f_{1} \geq 0, B=$ const $\geq 0, f_{1}^{\prime}(t, x, 0)=0$. 
Theorem 3.1. Suppose the following conditions hold:

1) supp $u_{0}(x)=[0,1] ; u_{0}(x) \leq P(1-x)^{\alpha}$ for $1-\varepsilon \leq x \leq 1, \alpha=$ const $\geq 1$, $P=$ const $>0, \varepsilon=$ const $>0$;

2) $g(t, x, u) \geq g_{l}(u), g_{l} \geq 0, g_{l} \in C, \quad \int^{1} d \xi / g_{1}(\xi)<+\infty$;

3) $A_{J}(t) \equiv P^{-/ / \alpha}\left(H^{-1}(t)\right)^{1 / \alpha}-B t \geq 0$ for small $t$, where $H(s) \equiv \int_{0}^{s} d \xi / g_{1}(\xi)$.

Then there exists such $t_{0}>0$ that $u(t, x)=0$ for $1-A_{1}(t) \leq x \leq 1,0 \leq t \leq t_{0}$.

Proof. Let us construct such a function $v_{0}(x)$ that $v_{0}(x)=P(1-x)^{\alpha}$ for $1-\varepsilon \leq x \leq 1$ and $v_{0}(x) \geq u_{0}(x)$ for other $x$. Let us consider a comparison function $w_{1}(t, x)$, defined by the relation

$$
\int_{w_{1}}^{v_{0}(x-B t)} d \xi / g_{1}(\xi)=t \text { for } \int_{0}^{v_{0}(x-B t)} d \xi_{/} / g_{1}(\xi) \geq t \text { and }
$$

equals to zero for other $t, x$. It is easy to see that $w_{1}(0, x) \geq u_{0}(x), L_{1} w_{1} \geq 0$ at the points where $w_{1}(t, x)$ is smooth and (1.3) is valid with $w_{1}$ instead of $u$. It follows with the aid of Theorem 1.1 that $u(t, x) \leq w_{1}(t, x)$. From the definition of $w_{1}(t, x)$ one has $w_{1}(t, x)=0$ for $H\left(v_{0}(x-B t)\right) \leq t$, in particular for $x-B t \geq 1-\varepsilon$ and $1-x+B t \leq\left[H^{-1}(t) / P\right]^{1 / \alpha}$. To finish the proof it remains to choose such $t_{0}$ that two previous inequalities are valid for $0 \leq t \leq t_{0}$.

Remark 3.1. If $A_{1}(t)=0$ for small $t$ then $u(t, x) \equiv 0$ for $x \geq 1$ and small $t:$ it follows from the proof of Theorem 3.1.

Theorem 3.2. Suppose the following conditions hold:

1) $g(t, x, s) \leq K s^{n}$ for small $s \geq 0,0<n<1, K=$ const $>0$;

2) supp $u_{0}(x)=[0,1], u_{0} \geq P(1-x)^{\alpha}, 1-\varepsilon \leq x \leq 1, \varepsilon>0, \alpha \geq 1, P=$ const $>0$;

3) $A_{2}(t) \equiv B t-P^{-t / \alpha}[t(K(1-n)+\delta)]^{I /(\alpha(t-n))} \geq 0$ for small $t$ and some $\delta>0$.

Then there exists such $t_{0}>0$ that $u(t, x)>0$ for $1+A_{2}(t) \geq x \geq 1, t \leq t_{0}$. 
Proof. Let us choose such a continuous in $\mathbf{R}$ and smooth for $x \neq 1$ function $v_{0}(x)$ that $v_{0}(x)=\left[P(1-x)^{\alpha}\right]^{1-n}$ for $1-\varepsilon \leq x \leq 1,0 \leq v_{0}(x) \leq u_{0}(x)^{1-n}$ for other $x$. Let us consider the function

$$
w_{2}(t, x)=\left\{\left[v_{0}(x-B t)-(K(1-n)+\delta) t\right]_{+}{ }^{1 /(1-n)} \equiv \lambda^{1 /(1-n)}\right.
$$

It is easy to see that (1.3) is valid for $w_{2}(t, x)$. Further, at the points where $w_{2}(t, x)$ is smooth one obtains $L_{1} w_{2}=-(1-n)^{-1} \lambda^{n /(1-n)}\left[v_{0}^{\prime} B+(K(1-n)+\delta]+\right.$ $f_{1}\left(t, x,\left(w_{2}\right)_{x}\right)+B(1-n)^{+1} \lambda^{n /(1-n)} v_{0}^{\prime}+g\left(t, x, \lambda^{1 /(1-n)}\right) \leq(1-n)^{-1} \lambda^{n /(1-n)} \times$ $\left[f_{1}\left(t, x,\left(w_{2}\right)_{x}\right) /\left(w_{2}\right)_{x}-\delta\right] \leq(1-n)^{-1} \lambda^{n /(1-n)}\left[f_{1}^{\prime \prime}\left(t, x,\left(w_{2}\right)_{x}\right)-\delta\right] \leq 0$, if $\varepsilon$ is small and $v_{0}(x)$ is suitably chosen.

The function $w_{2}(t, x)$ is positive if $v_{0}(x-B t)>(K(1-n)+\delta) t$, in particular for $1-\varepsilon+B t \leq x \leq 1+B t$ and for $x<1+B t-P^{-1 / \alpha}(K(1-n)+$ $\delta)^{1 / \alpha(1-n)\}} t^{1 / \alpha(1-n)\}}$. Applying Theorem 1.1 on gets the required result.

Remark 3.2 If $A_{2}(t)=0$ for small $t$ then $u(t, x)>0$ for $1-\varepsilon \leq x \leq 1, \varepsilon>0$ and small $t$ : it follows from the proof of Theorem 3.2.

Remark 3.3. Suppose $g(t, x, s) \equiv K|s|^{n-1} s, 0<n<1, K=$ const $>0$, $u_{0}(x)=P(1-x)^{\alpha}$ for $1-\varepsilon \leq x \leq 1, \alpha \geq 1, P=$ const $>0, \varepsilon>0$. then the following statements follow from Theorems 3.1, 3.2:

1) If $\alpha(1-n)>1$ then sup supp $u(t, x)<1$ for small $t>0$.

$$
x
$$

2) If $\alpha(1-n)<1$ then sup supp $u(t, x)>1$ for small $t>0$.

3) Suppose $\alpha(1-n)=1$; then:

a) If $B<K P^{-1 / \alpha}(1-n)$ then sup supp $u(t, x)<1$ for small $t>0$; $x$

b) If $B>K P^{-1 / \alpha}(1-n)$ then sup supp $u(t, x)>1$ for small $t>0$; 


\section{References}

[1] BARLES, G. Asymptotic behaviour of viscosity solution of first order HamiltonJacobi equations. Ric. mat., 1985, v. 34, 2, p. 227-260.

[2] CONWAY, E.D. and HOPF, E. Hamilton's theory and generalized solutions of the Hamilton-Jacobi equation. J. Math. and Mech., 1964, v. 13, 6, p. 939-986.

[3] CRANDALL, M.G. and LIONS, P.L. Hamilton-Jacobi equations in infinite dimensions 1. Uniqueness of viscosity solutions. J. Func. Anal., 1985, v. 62, 3, p. 379-396.

[4] CRANDALL, M.G. and LIONS, P.L. Hamilton-Jacobi equations in infinite dimensions 2. Existence of viscosity solutions. J. Func. Anal., 1986, v. 65, 3, p. 368-405.

[5] CRANDALL, M.G. and LIONS, P.L. Hamilton-Jacobi equations in infinite dimensions 3. J. Func, Anal., 1986, v. 68, 2, p. 214-247.

[6] CRANDALL, M.G., EVANS, L.C. and LIONS, P.L. Some properties of viscosity solutions of Hamilton-Jacobi equations. Trans. Amer. Math. Soc., 1984, v. 282,2 , p. 487-502.

[7] CRANDALL, M.G. and LIONS, P.L. Viscosity solutions of Hamilton-Jacobi equations. Trans. Amer. Math. Soc., 1983, v. 277, 1, p. 1-42.

[8] KRUZSHKOV, S.N. Generalized solutions of nonlinear first order equations in several spaces variables I. Matem. Sbornik (USSR), 1966, v. 70, 3, p. 394415 .

[9] KRUZSHKOV, S.N. Generalized solutions of nonlinear first order equations in several spaces variables 2. Matem. Sbornik (USSR), 1967, v. 72, 1, p. 108134.

[10] KRUZSHKOV, S.N. Generalized solutions to Hamilton-Jacobi equations of euconal type 1. Problems, existence, uniqueness and stability, some properties of the solutions, Matem. Sbornik (USSR), 1975, v. 98, 3, p. 450-493.

[11] KRUZSHKOV, S.N. On the minimax representation of the solutions to nonlinear first order equations. Funk. Analiz. (USSR), 1969, v. 3, 2, p. 57-66.

[12] RYKOV, Y.G. On the small time behaviour of the generalized solutions to nonlinear first order equations. Classical and nonclassical boundary problems to partial differential equations. Reports of the conference on differential equations, Samara, USSR, 1987, p. 125. 
[13] RYKOV, Y.G. On the decrease for large time of the generalized solutions of Cauchy problem to Hamilton-Jacobi equations. Uspekhi mat. nauk (USSR), 1987 , v. 42,4 , p. $155-156$. 